\section{Mesenteric Artery Pseudo- aneurysm: A Rare and Complex Complication of Abdominal Surgery}

Sir,

A 27-year female was operated for enteric perforation with an ileostomy formation, in a basic health unit in periphery. Two months after the initial surgery, she presented with non-specific abdominal pain and burst abdomen. On examination, she was pale, had tachycardia (104/min), and mild fever $\left(37.8^{\circ} \mathrm{C}\right)$. She had an infected weeping midline abdominal surgical wound. Laboratory tests showed moderate anaemia (haemoglobin $7.5 \mathrm{~g} / \mathrm{dl}$ ) and hypokalemia (potassium $3.14 \mathrm{mmol} / \mathrm{L}$ ). Contrast enhanced computed tomography revealed an outpouching of contrast in communication with the branch of superior mesenteric artery (SMA), representing pseudo-aneurysm (Figure 1). An ileostomy in right iliac fossa and a wide midline gaping surgical wound (burst abdomen) was also identified (Figure 2). A fluid collection was also visualised in right para-colic gutter extending from the pseudo-aneurysm (Figure 3).

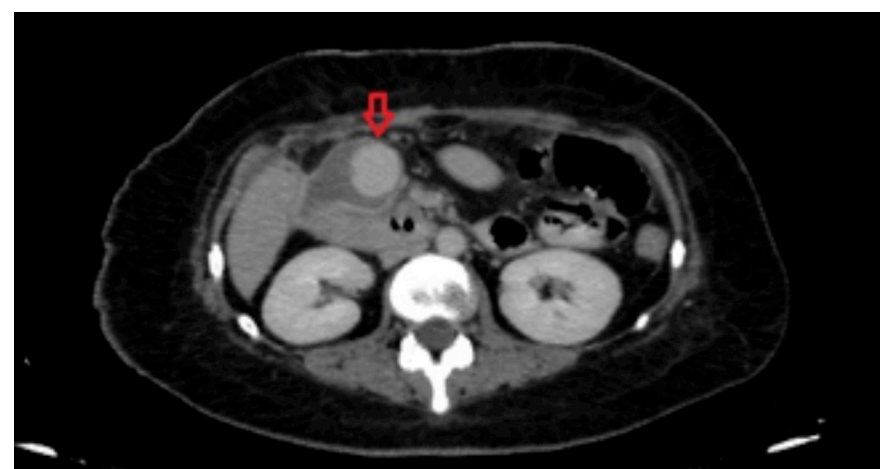

Figure 1: Contrast filled outpouch in communication with branch of superior mesenteric artery, and surrounding fluid collection (red arrow).

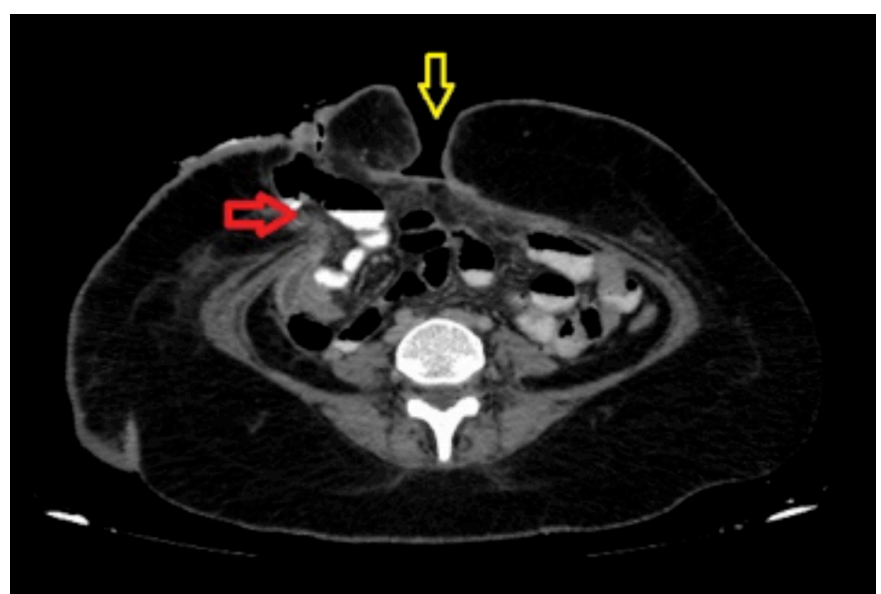

Figure 2: Right iliac fossa ileostomy (red arrow), and midline open surgical wound (yellow arrow).

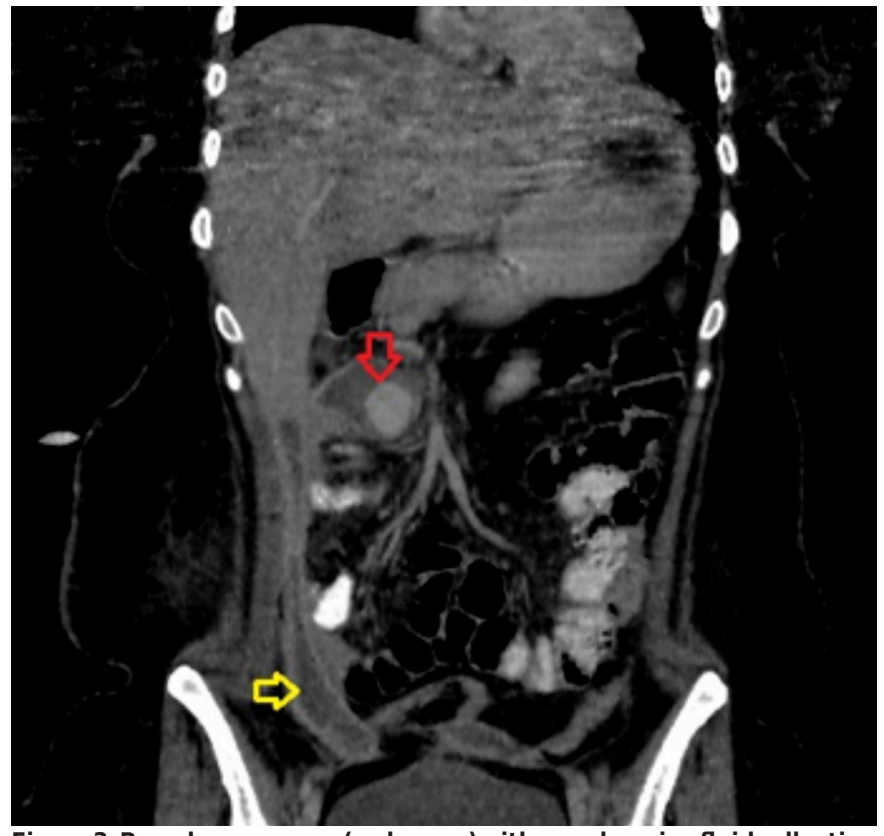

Figure 3: Pseudo-aneurysm (red arrow) with an enhancing fluid collection extending from pseudo-aneurysm in right para-colic gutter (yellow arrow).

Patient was re-explored. Peroperatively, a bleeding pseudo-aneurysm from the branch of SMA was confirmed, which was ligated. Approximately 500cc of serosanguineous fluid was drained from right para-colic gutter and a surgical drain was placed. Ileostomy stoma was revised and colon was brought out as mucous fistula. Postoperatively, packed red cells were transfused, potassium was replaced, broad-spectrum antibiotics and parenteral intravenous Ringer's lactate were administered. Oral fluids were started on $4^{\text {th }}$ postoperative day; and she was discharged on $6^{\text {th }}$ postoperative day.

Visceral artery aneurysms and pseudo-aneurysms are a rare entity, representing $0.1-0.2 \%$ of all vascular aneurysms; and found in $0.1 \%$ of autopsies. ${ }^{1,2}$ SMA pseudo-aneurysm is the rarest, and is caused by pancreatitis, infection, inflammation, post-traumatic dissection or post-surgical complication. ${ }^{3,4}$ This case most probably was related to the previous abdominal surgery.

Early diagnosis and timely treatment of this potentially fatal condition are warranted, which could be treated by angiographic embolisation and endovascular stent graft placement or by traditional open surgery. ${ }^{5}$ Mortality of endovascular technique is 12 to $33 \%$ as compared to 16 to $50 \%$ morbidity, associated with open surgical technique. ${ }^{5}$

Vascular pseudo-aneurysm is a rare postoperative complication, which could be catastrophic if not timely treated, either by open ligation or by angiographic endovascular embolisation, depending upon the etiology of pseudoaneurysm and the surgeon's expertise.

\section{CONFLICT OF INTEREST:}

The authors declared no conflict of interest. 


\section{AUTHORS' CONTRIBUTION:}

AS: Study design, analyses of all radiological, and clinical data, and manuscript writing.

HS: Patient care, of surgical management of the patient, clinical data collection, and manuscript writing.

SSAS: Concept of study, surgical management of the patient, clinical data collection and manuscript writing.

\section{REFERENCES}

1. Olsen $A B$, Ralhan $T$, Harris Jr JH, Evani V. Superior mesenteric artery pseudoaneurysm after blunt abdominal trauma. Ann Vasc Surg 2013; 27(5):674-8. doi: 10.1016/j. avsg.2012.09.006.

2. Abdelgabar A, d'Archambeau O, Maes J, Van den Brande F, Cools P, Rutsaert RR. Visceral artery pseudoaneurysms: Two case reports and a review of the literature. J Med Case Rep 2017; 11(1):126. doi.org/10.1186/s13256- 017-12 91-6.

3. Guirgis M, Xu JH, Kaard A, Mwipatayi BP. Spontaneous superior mesenteric artery branch pseudoaneurysm: A rare case report. EJVES Short Rep 2017; 37:1-4. doi: 10.1016/j.ejvssr.2017.09.001.

4. Kaczynski J. Superior mesenteric artery branch pseudoaneurysm mimicking an acute appendicitis. BMJ
Case Rep 2012; 2012:bcr0220125826. doi.org/10.1136/ bcr.02.2012.5826.

5. Cowan S, Kahn MB, Bonn J, Becker GJ, DiMuzio P, Leichter $R$, et al. Superior mesenteric artery pseudoaneurysm successfully treated with polytetrafluoroethylene covered stent. J Vasc Surg 2002; 35(4):805-7. doi.org/10.1067/ mva.2002.121754.

Aniqua Saleem ${ }^{1}$, Hira Saleem ${ }^{2}$ and Sharjeel Sarfraz Ahmed Sheikh ${ }^{3}$

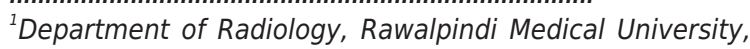
Rawalpindi, Pakistan

${ }^{2}$ Department of Surgery, Shifa International Hospital, Islamabad, Pakistan

${ }^{3}$ Department of Surgery, Rawalpindi Medical University, Rawalpindi, Pakistan

Correspondence to: Dr. Aniqua Saleem, Department of Radiology, Rawalpindi Medical University, Rawalpindi, Pakistan E-mail: aneeqa_068@hotmail.com

Received: January 18, 2021; Revised: February 21, 2021; Accepted: April 24, 2021

DOI: https://doi.org/10.29271/jcpsp.2022.01.128 